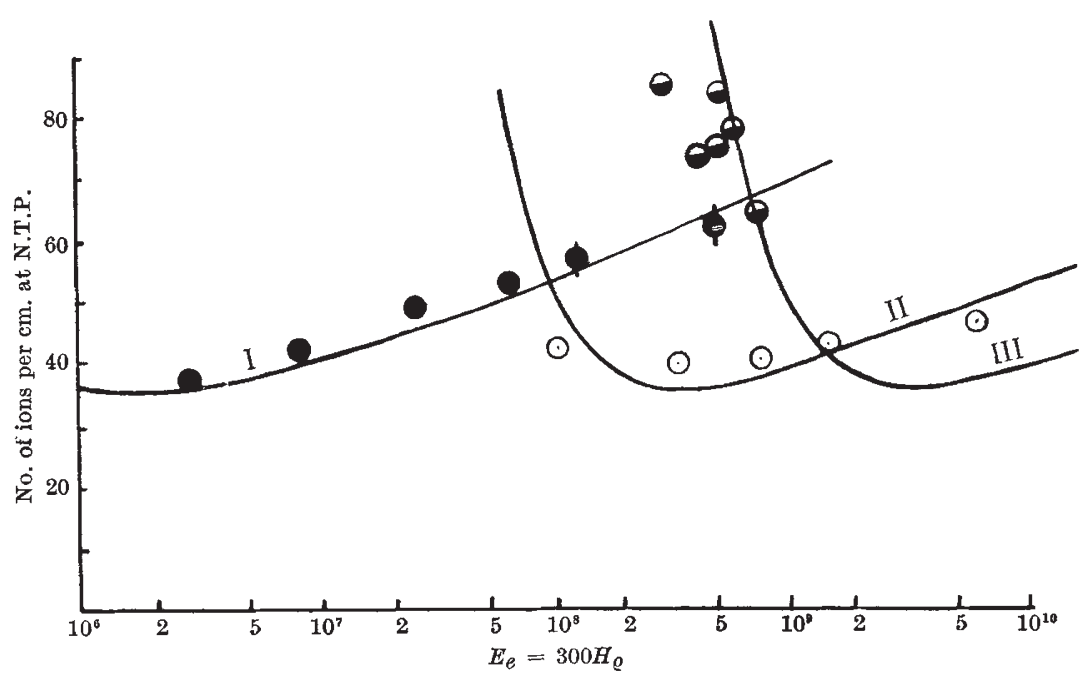

ENERGy IONIZATION CURVES FOR EEECTRONS (I), MESONS (II), PROTONS (III).

common anode placed between two equal cathodes at the same potential but at variable spacing has been extensively investigated experimentally, G. E. Gardam ${ }^{1}$ appears to be the first to attempt a theoretical treatment. He showed that the ratio of the currents flowing to each cathode can be calculated in terms of the dependence of the cathode fall on the current density, the conductivity of the electrolyte, and the electrode spacings, for the case of a uniform field corresponding with the experimental arrangement of Haring and Blum ${ }^{2}$. This theory also predicts the relation between the cathode currents and the throwing power ${ }^{3}$, which latter is a function of the ratio of the weights of the deposits and the electrode distances. A good

$$
\begin{aligned}
& 0=\text { ELECTRON } \\
& O=\text { MESON }(M=170 \mathrm{~m}) \\
& \ominus=\text { PROTON }
\end{aligned}
$$

observed range, that is, between $2 \times 10^{6} \mathrm{ev}$. and $5 \times 10^{8}$ ev., both as regards absolute value and variation with energy.

For mesons, the agreement with theory is not as good. However, the results do show a small rise of ionization with energy, though not as much as is expected. Possibly the errors of the curvature measurements may be the cause of this discrepancy. Since the protons above $2 \times 10^{3} \mathrm{ev}$. ionize less than mesons of the corresponding energy, the relatively low observed value in the highest energy group may possibly be explained as due to some protons being included in the group. Unfortunately, no meson track was observed with a momentum low enough to allow a reliable mass determination.

Among 3,000 tracks, eight recognizable proton tracks (all positive) have been observed. The observed values of the mean specific ionization on each individual proton track are shown as half-shaded circles, and show a satisfactory agreement with the theoretical curve. No alpha particles or other multi-charged particles were observed, nor any deuterons.

A detailed account of the investigation will be published elsewhere.

Physical Laboratories,

University of Manchester.

R. L. Sen Gưta. June 6.

${ }^{1}$ Corson and Brode, Phys. Rev., 53, 773 (1938).

\section{Current Distribution and Throwing Power in Electrolytes}

THERE are two interesting aspects of the distribution of a direct current flowing between soluble metal electrodes in an aqueous electrolyte confined in an insulating vessel. Theoretically the problem appears to be soluble, ideally at least, by classical potential theory using the electrochemical theory of the anode and cathode fall. The distribution is of great practical importance in attaining uniform deposits on curved surfaces and in holes, and in avoiding heavy deposits on sharp edges and projections.

Although the current distribution between a agreement is reached with both his own experimental results and those of other investigators.

Recently the current distribution in electrolytes for various electrode systems giving non-uniform fields has been examined theoretically by C. Kasper ${ }^{4}$ by applying potential theory; the important effects of potential falls at the electrodes are, however, neglected.

A new theoretical and experimental investigation of the current distribution between plane electrodes at variable spacings in a non-uniform field has been carried out in an attempt to remedy this defect. The experimental arrangement consists of an anode, a main cathode with a hole in the centre from which, on the side opposite to the anode, a rubber tube extends, ending in a small subsidiary cathode (at the same potential as the main cathode). The current to the subsidiary electrode is calculated approximately from the sum of two potential terms. The first is the potential at the centre of the hole determined by the potential field between the three electrodes; this field problem is similar to that in a cathode-ray oscillograph ${ }^{5}$. The second is equal to the difference between the two cathode falls. Allow. ance is made for the resistance of the electrolyte between the two cathodes. Assuming the usual logarithmic relation between the cathode fall and the current density, it is found that the ratio of the subsidiary to the main cathode current decreases with increasing distance between the cathodes and with increasing current to the main cathode, the rate of change being very rapid at small spacings. Furthermore, this ratio is independent of the distance between the anode and the main cathode over a wide range.

Using copper electrodes in neutral and acid solutions of copper sulphate, these theoretical predictions are found to be satisfactorily confirmed by the present experiments, in which measurements of the electrode potential fall by means of probe electrodes are introduced. A detailed report of these investigations will be published later. A. v. ENGEL.

Inorganic and Physical Chemistry Department,

Imperial College of Science and Technology, London, S.W.7. May 28.

1 Gardam, G. E., Trans. Far. Soc., 34, 698 (1938). Haring, H. E., and Blum, W., Trans. Amer. Electrochem. Soc., 44,

' Field, S., and Weill, A. D., "Electro-Plating”' (London, 1938).

4 Kasper, C., Amer. Electrochem. Soc., ry, preprint 28 (April 1940). ' Ollendorff, F., "Potentialfelder der Elektrotechnik" (Berlin : Springer, 1932). 